\title{
Refinement the form and parameters of quantum space of the universe
}

\author{
Valentyn Alexeevich Nastasenko*
}

Professor, Department of Transport Technologies, Kherson State Maritime Academy, 141, Ushakovast. 41, 73003, Kherson, Ukraine

\section{Article Info}

*Corresponding author:
Valentyn Alexeevich Nastasenko
Professor
Department of Transport Technologies
Kherson State Maritime Academy
141, Ushakovast. 41, 73003
Kherson, Ukraine
Tel: +38(050) 807-91-99
E-mail: nastasenko2004@ukr.net

Received: February 26, 2019

Accepted: March 5, 2019

Published: March 11, 2019

Citation: Nastasenko VA. Refinement the form and parameters of quantum space of the universe. Int I Cosmol Astron Astrophys. 2019; 1(2): 62-66.

doi: 10.18689/ijcaa-1000115

Copyright: ( $\subset 2019$ The Author(s). This work is licensed under a Creative Commons Attribution 4.0 International License, which permits unrestricted use, distribution, and reproduction in any medium, provided the original work is properly cited.

Published by Madridge Publishers

\begin{abstract}
The refinement of the parameters of the quantum space of the Universe was performed on the basis of previous works. However, this problem was not fully resolved, since the previously found elementary quantum of space in the form of a straight-sided prism did not correspond to the principle of minimalistic of the parameters of its shape. A new shape of the elementary quantum of the Universe in the form of a trihedral prism is proposed. This quantum is supplemented by its antipode, which has a mirror shape. In a connected form, they constitute a pair - a dipole. A new circular quantum of the Universe space is formed from 6 such dipole pairs. Strong interconnection of neighboring quanta is ensured by the possibility of mutual penetration of their neighboring edges into each other, which follows from the Heisenberg uncertainty principle. The layering of 12 circular quanta on the top of each other provides them with a helical shape with a complete circular along their periphery, which reduces them to a mini-helix. New numerical values for the basic parameters of quanta and their interrelations are given. A new form of quanta and their new properties are of great importance for understanding the fundamentals of the material world. It has especially great significance for philosophy development, quantum physics, physics of elementary particles and cosmology.
\end{abstract}

Keywords: Quantum of the Universe space; Form and sizes; Parameters of gravitational field; Quantum physics

\section{Introduction}

The infinite space of the Universe has an internal structure, the limiting state of which is the quanta of minimum size. Argumentation of quantum of space parameters of the Universe is a complicated scientific problem, as its reliable information is unknown, except for its general characteristics -quantum should be minimum and indivisible. Its definition had a great significance for the majority of top-level world scientists throughout history of civilization [1].

Currently, the most complete solution to this problem has been proposed in [2], where is it divided into initial elementary quanta and final circular hexahonal shaped quanta composed of them. However, a number of initial scientific propositions and solutions proposed in [2] do not fully correspond to the principles of minimizing the parameters of an elementary quantum.

Elimination of these drawbacks is the main goal of the work performed. Her scientific novelty is the refinement of the parameters new form of an elementary quantum on the basis of strict physical laws and principles, which led to a new form of a finite circular quantum and made it possible to single out its new properties for the first time. A new form of quanta and their new properties are of great importance for understanding the fundamentals of the material world. It has especially great significance for philosophy 
development, quantum physics, physics of elementary particles and cosmology. Solvation of the given problem constitutes the main aim of the executed work. Strict argumentation and definition of possible real shape and parameters of quantum of the Universe space, based on the indubitable principles of physics, constitute its scientific novelty.

\section{Analysis of problem state, problem formulation and search of the lines of approach its solution}

In work [2] it was shown that the solution of the problem of determining the minimum quantum of the Universe space is reduced to the scientific discovery [3], since the parameters and form of the quantum are unknown and have no obvious analogues. In the framework of general principles solution of scientific-technical problems [4], the original task was divided into two subtasks:

\section{A - definition of quantum of space form \\ B - definition of quantum of space parameters.}

The form definition of quantum in work [2] was based on work [5] and reduced to (Figure 1).

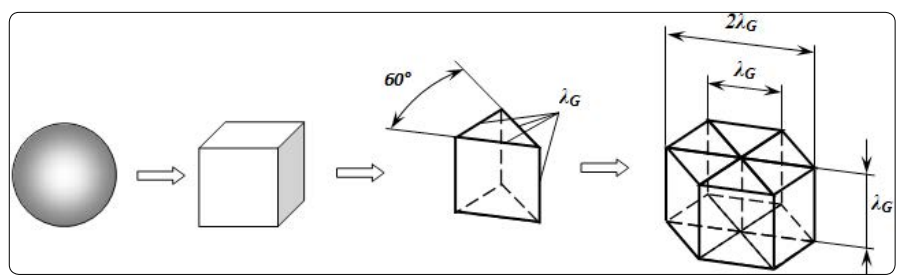

Figure 1. Concept of quantum of space of the Universe

To eliminate this drawback, in work [2], the determination of the parameters of the minimum quantum of Universe space was reduced to the wave parameters of the gravitational field, since they characterize real values of the oscillation frequency $v_{G}(1)$ and wavelength $\lambda_{G}(2)[6]$ :

$$
\begin{aligned}
& v_{G}=v_{p}=\sqrt{\frac{c^{5}}{G h}}=7.39994 \cdot 10^{42}\left(s^{-1}\right) \rightarrow 7.4 \cdot 10^{42}\left(s^{-1}\right) . \\
& \lambda_{G}=\frac{c}{v_{G}}=4.051249|432| \cdot 10^{-35}(\mathrm{~m}) .
\end{aligned}
$$

Where $G$ - gravitational constant:

$$
G=6.67408(31) \cdot 10^{-11} \frac{\mathrm{m}^{3}}{\mathrm{~kg} \cdot \mathrm{s}^{2}} ;
$$

$c$ - speed of light in vacuum:

$c=0.299792458($ exactly $) \cdot 10^{9} \frac{\mathrm{m}}{\mathrm{s}}$

h- Planck's constant:

$h=6.626070040(81) \cdot 10^{-34} \mathrm{~J} \cdot \mathrm{s}=6.26070040(81) \cdot 10^{-34} \frac{\mathrm{kg} \cdot \mathrm{m}^{2}}{\mathrm{~s}}$.

Magnitude and accuracy of parameters $G, c, h$ is recommended CODATA [7]. Nevertheless, the further analysis of the found structure of elementary quanta and circular quantum of space figure 1 has shown that they also do not match the criteria of the minimalistic, and, thus, require more precise definition, that is performed in the given work.

\section{Argumentation of the new shape of quantum of space of the Universe}

Further criteria for the minimization of elementary quanta are associated with trihedral rectilinear prisms that form a hexagonal circular quantum of the space of the Universe (Figure 1). The ends (flank) of this elementary quanta form a square with edges whose length is $\lambda_{G}$. However, the square in this ends doesn't comply with the minimal principle, as the simplest form is a triangle.

In this work is offered with the substitution of square on the flank of prism with regular triangle. This is transforming the elementary quantum to prism of a new shape figure 2 , in which the triangle faces on flank have the length $\lambda_{G^{\prime}}$ and their tilting angle constitutes value $60^{\circ}$. As the parental altitude of ribs that are opposite to new triangle face of elementary quanta, is bigger than altitudes of their trihedral, this leads to nonparallel misalignment of the upper rib and quantum basis. Its results - in nonparallel upper and lower layers of circular quanta, that alters space of the Universe, which is contrary to astronomical observations.

Eliminating the indicated disadvantage of new quanta is made possible by the substitution of straight parental altitude of rib, of the opposite to triangular of flank face, on an arc, that is having the radius $\lambda_{G}$ with arc length that is equal to value $\lambda_{G}$. This is equal to the value of a radian.

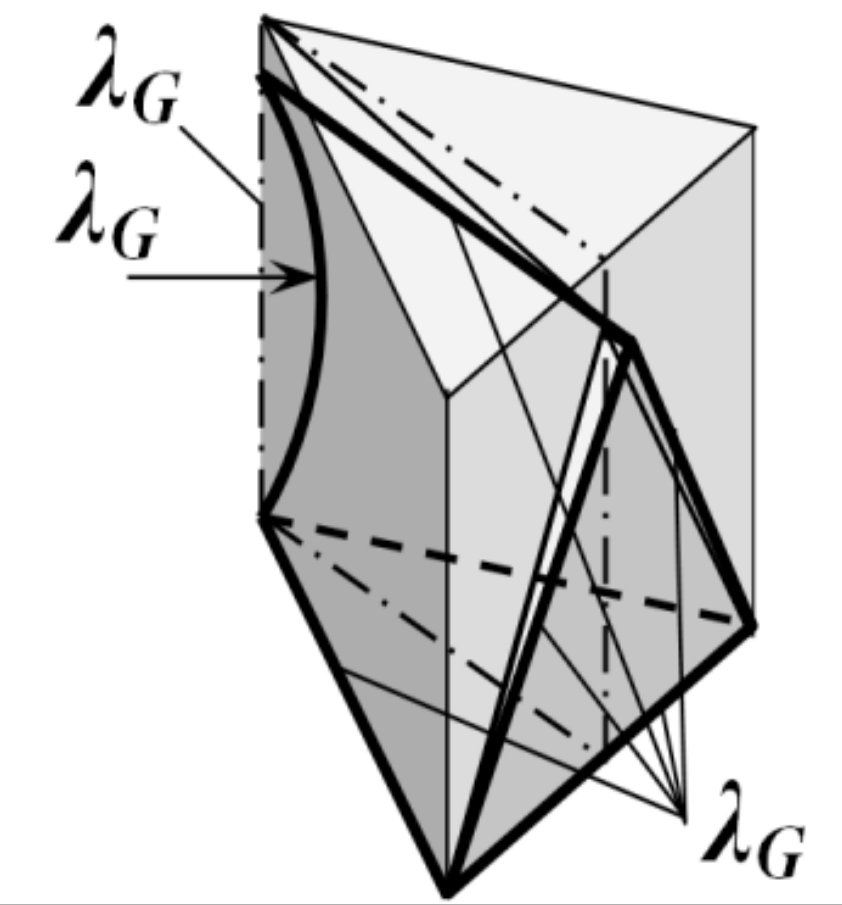

Figure 2. Transformation of parental elementary quantum into the new one

It should be taken into consideration that for completeness of tight packing, parental elementary quantum should have antipode in the form of a mirror image with common radius rib for all these quanta (Figure 3a,b). Formation of elementary quanta with antipode into a overall quantum (dipole) is possible at compression of face square of the cubic parental quantum into a rhomb, in which the lesser diagonal is equal 
to value $\lambda_{G}$ (Figure $3 c$ ). Six pairs of new elementary quantumdipoles - form a new circular quantum of space of the Universe, having 12 side hexagonal faces (Figure 3d). General structural scheme of their transformation and combination is shown in (Figure 3).
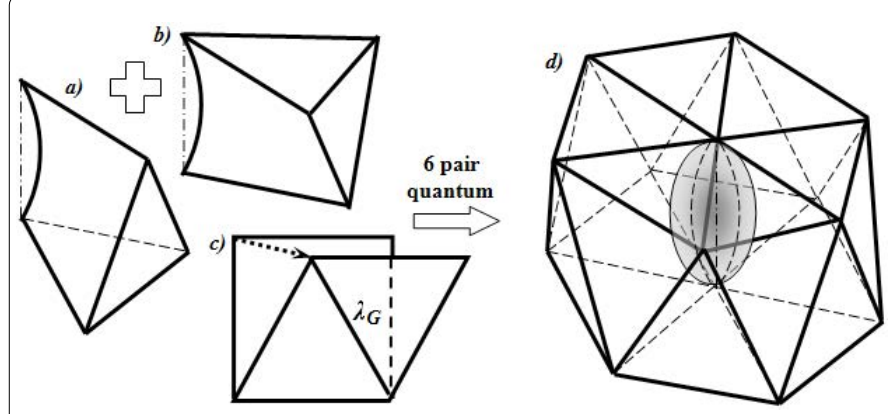

Figure 3. New image of a quantum of space of the Universe and scheme of its formation: a) elementary quantum, b) antipode, c) deformation of a square in a rhombus, d) new circular quantum

The arc ribs of dipoles form a solid (nucleus) made up of six arcs of radius $\lambda_{G}$ on the central axes of circular quantum, which closes compose a circle and goes absolutely with Planck's constant $h$ and six circular Planck's constants $\hbar$, the correlation of which constitutes value $2 \pi$. In this connection nucleus is the inner structure of the circular quantum and cannot exist separately. Gravitational radius of circular quantum is connected with it nucleus.

Stable limiting separating planes in elementary and circular quanta of space is no, as dislocation along them is impossible, and ribs, motion along which is possible, in the framework of Heisenberg uncertainty principle [8], have blurred configurations (spot), interpenetrating each (Figure 4).

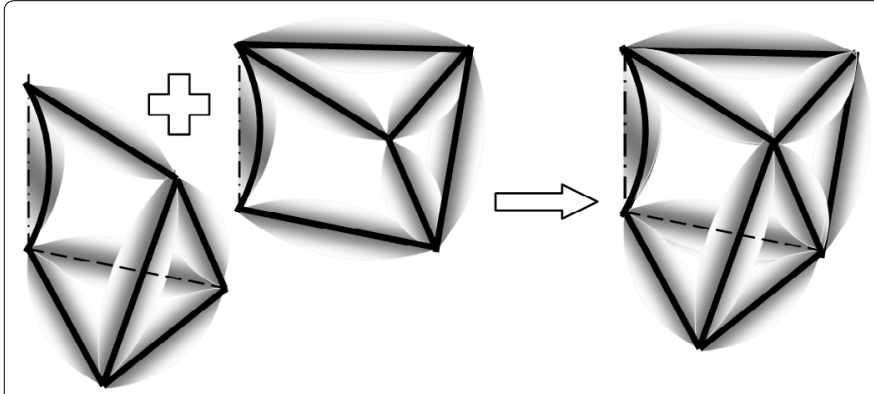

Figure 4. Formation of ribs of elementary quantum (a), antipode (b) and paired quantum - dipole (c) in the framework of Heisenberg uncertainty principle

Despite of angular difference of upper and lower subspaces of quantum, they pack in a good way in the frameworks of blurred form of their faces and interpenetration of their ribs figure 5 onto value $\pm \Delta \lambda_{G}(3)$ :

$\pm \Delta \lambda_{G}= \pm \lambda_{G}\left(1-\cos 30^{\circ}\right)= \pm 4.051249|432|$.

$10^{-35}(\mathrm{~m}) \times\left(1-\cos 30^{\circ}\right)= \pm 0.5427645069 \cdot 10^{-35}(\mathrm{~m})$.

In this case, the limits of minimal blurring the ribs are equal to level (4):

$\delta=\frac{ \pm \Delta \lambda_{G}}{\lambda_{G}}=\frac{ \pm 0.5427645069 \cdot 10^{-35}(\mathrm{~m})}{4.051249|432| \cdot 10^{-35}(\mathrm{~m})} \times 100 \%= \pm 13.397 \%$.

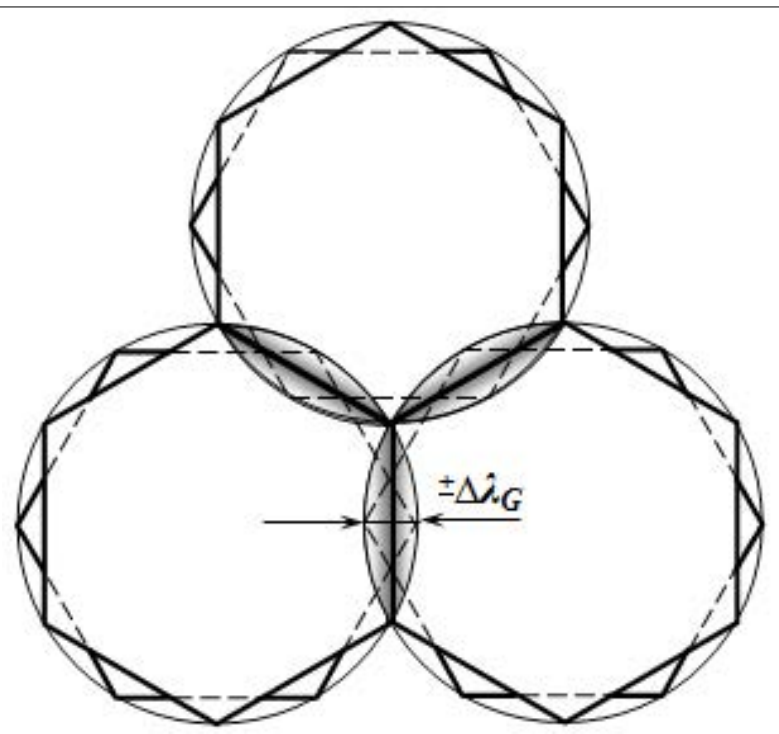

Figure 5. Packing of spiral quanta with each other in the interpenetration zones $\pm \Delta \lambda_{G}$

Due to this interpenetration, the grouping of circular quanta is not beyond the scope of structure, shown in figure 3 , and has strength of compounds. That is why a dodecahedral circular quantum is actually included into a hexagonal one, and their interpenetration $\pm \Delta \lambda_{G}$ intensifies of their compounds.

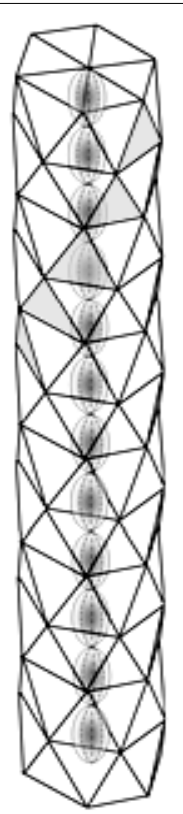

Figure 6. Formation of a complete turn of mini strings circular quanta on their 12 layers

Obliquity of side ribs on the elementary and circular quanta of space forms a complete spiral turn for packing of 12 layers of circular quanta placed one upon another (Figure 6). They include the inner central thread of the gravitational radii and the outer helical mini string, one turn of which is highlighted in gray. But what mainly the complete turning and wave $\lambda_{G 12}$ out of 12 circular quanta can be connected with shall still be found out. At the same time, despite the fact that the real altitude between the layers of circular quanta, that are lesser than value $\lambda_{G^{\prime}}$ it is possible to get on them, only having passed the complete path ribs $\lambda_{G^{\prime}}$ that do not violate quantization principles. 
The received spiral structure can be evidence of rotation of circular quantum's of space and the whole Universe, that dynamically increase their stability. Found out due to such kind of construction threads and winding mini strings can be the evidence of their presence in the Universe. Here with, direction of turns can be both right and left, though only one direction should be dominating, and the second should be stock.

The received structure of the new circular quantum of space of the Universe of Planck's value (3) that is shown in figure 6, is the closest to the ideal one, both in shape and according to prerequisites to quantization. For winding structures and their turnover figure 6 - finishing of principles is still required.

Possible variants of rotation the quanta are shown in (Figure 7). Analysis of the system (a) showed that the rotation of quanta between themselves evoke doubts (?), since it leads to mutually opposite directions, which cause resistance in zones of mutual penetration of quanta. This requires energy, and its transformation leads to heat generation and heating of the Universe, which contradicts astronomical observations. However, cooperative rotation of quanta system layers (b) within the framework of the Universe model [9] is possible, which confirms this model and may indicate the presence of rotation axis in it.
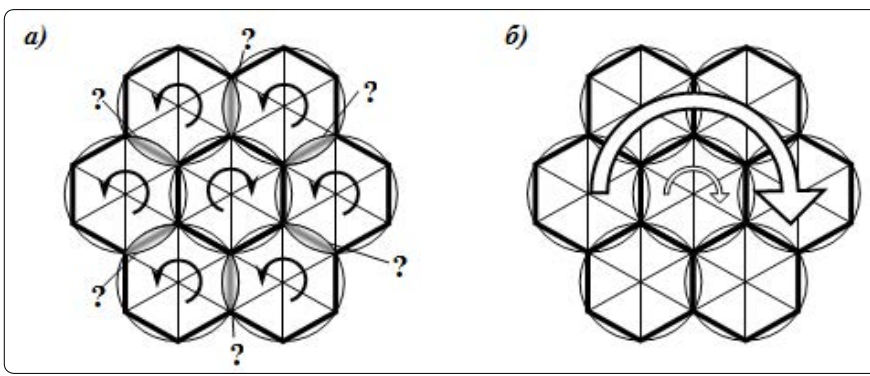

Figure 7. Structure of the mini strings and rotation options of the Universe space quanta

But location of rotation axis and answer the question of why it cannot pass through any other quantum mini string of the Universe still requires proof, including as a result of astronomical observations.

\section{Conclusion}

1. Parameters of all minimal quanta of space in the Universe are received by way of logical reasoning on the grounds of common principles of the material world structuring and scientific knowledge development from simple to the complicated, which do not contradict to the known laws of nature.

2. Minimal sizes of quantum of space are connected with wavelength $\lambda_{G}$ of the gravitational field which having the frequency $v_{G}=7.4 \cdot 10^{42} \mathrm{~s}^{-1}$ (constant Nastasenko) and speed $c$ of light in vacuum:

$\lambda_{G}=\frac{c}{v_{G}}=4.051249|432| \cdot 10^{-35}(\mathrm{~m})$.

3. The condition of quanta form minimization complies with elementary quanta in the shape of prisms with regular triangle basis and ribs length $\lambda_{G^{\prime}}$ which highlights their connection with quarks.
4. The condition of form minimization of flank sides of elementary quanta also complies with regular triangles; however, the opposite to face triangle rib is contracted into the sector of arc radius and length $\lambda_{G}$ that corresponds to radian value.

5. Elementary quantum of space has an antipode of mirror image bound to parental elementary quantum into a paired structure (dipole), which having arcs ribs, opposite to flank triangles, are common.

6. Formation of elementary quantum of space and its mirror antipode is possible from straight-sided trihedral prism at winding compression of its square flank into a rhomb and formation of cross connection along rhomb lesser diagonal with length $\lambda_{G}$.

7. Circular grouping of six pairs of elementary quanta of space and their antipodes, closely adherent to each other with their side faces with common central axes of their arc ribs, forms the circular quantum of space of the Universe in the shape of a prism, consisting of twelve triangle faces.

8. Jumps in elementary and circular quanta are possible along ribs only, and is impossible along diagonals of square ones and altitudes of triangle faces, insofar as to which they do not have stable limiting faces.

9. Ribs in quanta of space, in the frameworks of Heisenberg uncertainty principle, have blurred configurations (spot), interpenetrating each other for a depth of $\Delta \lambda_{G}$ :

$$
\pm \Delta \lambda_{G}= \pm \lambda_{G}\left(1-\cos 30^{\circ}\right)= \pm 0.5427645069 \cdot 10^{-35}(\mathrm{~m}) .
$$

10. Arc ribs of all paired quanta of space - dipoles - form a solid (nucleus), consisting of 6 arcs of radius $\lambda_{G^{\prime}}$ that closes a complete circle and goes exactly with Planck's constant $h$ and six circular Planck's constants $\hbar$, correlation between which constitutes value $2 \pi$, on the central axe of circular quantum.

11. Nucleus of circular quantum is its inner structure, with which gravitational radius is connected and it cannot exist separately from the circular quantum.

12. Obliquity of side ribs on quanta of space forms the complete spiral turn of packing, consisting of 12 layers of circular quanta, placed one upon the other, that is the evidence of turning of circular quanta and the whole Universe, that dynamically increases their stability. Though, what mainly the complete turning and wave $\lambda_{G 12}$ out of 12 circular quanta can be connected with and preconditions to their formation and turning shall still be found out.

\section{References}

1. Vasilyeva VV, Krotovai AA, Bull DV. History of Philosophy: Textbook for universities. M.: Academic Project: 2005.

2. Nastasenko VA. Definition form and Parameters of quantum of space of the Universe. Int J CosmolAstronAstrophys. 2019 1(2): 42-45. doi: 10.18689/ijcaa-1000111

3. Nastasenko VA. Methodology for solving creative tasks high level of complexity III. 2016; 4(48): 53-59. 
4. You are the only citizen of the world (Ukraine). The law of Ukraine. Kiev: School. 2003; 142.

5. Nastasenko VA. Ground of parameters minimum quantum of space of universe: Scientific Bulletin KhDMA. 2012; 1(6): 285-297.

6. Nastasenko VA. Physical-Mathematical Method Defined of Wave Parameters Gravitation Field. Int J Eng Tech. 2019; 11(1): 62-64. doi: 10.7763/IJET.2019.V11.1124
7. CODATA Internationally recommended values of the Fundamental Physical Constants. NIST reference.

8. Alekseev DV, Bonch-Bruevich AM, Voronov-Romanov AS, et al. Physical encyclopedia hessky dictionary. Pod red. AM Prohorov (Ed.). Moskva :Sov. Encyclopedia.1983; 465: 826-33.

9. Nastasenko VA. New model of Universe birth - our answer. Materials of international practical conference. Kherson, KHDMA, 2015. 